\title{
E-MRS Holds Fourth Annual Spring Meeting in Strasbourg
}

The European Materials Research Society (E-MRS) held its Fourth Annual Spring Meeting in Strasbourg. France, at the Council of Europe facility during the week of June 16, 1986. The meeting was composed of four technical symposia planned well in advance by symposium chairs on behalf of E-MRS. A fifth symposium was added quite late to the overall program and complemented the program by bringing additional attendees and topics to the meeting. The five topical areas covered included advanced materials for telecommunications, (Symposium A), laser processing and diagnostics (Symposium B), state of the art of computer simulation of casting and solidification processes (Symposium C), dielectric layers in semiconductors: novel technologies and devices (Symposium D), and the late edition, magnetic thin films (Symposium E). E-MRS' ability to add an entirely new symposium at the last minute reflects the flexibility and responsiveness for which MRS-style societies strive.

Approximately 300 participants filled the lecture rooms throughout the week. The meeting began on Tuesday, June 17 with an all-society morning plenary session. A welcoming_address by J.P. Massué, Scientific Advisor to the Council of Europe's Parliamentary Assembly was followed by Nobel laureate Sir Nevill F. Mott, who spoke on oxidation of silicon. Mott, who recently attended a conference in Paris on this subject, reported that the underlying microscopic mechanisms responsible for the various stages of oxidation of silicon both by dry oxygen and by water vapor are still not clear. Questions on the role of moving chemical species and electrical considerations still arise. Both in the linear, or very thin film, regime and in the thicker parabolic rate law regime, experiments are in progress to elucidate the precise mechan-

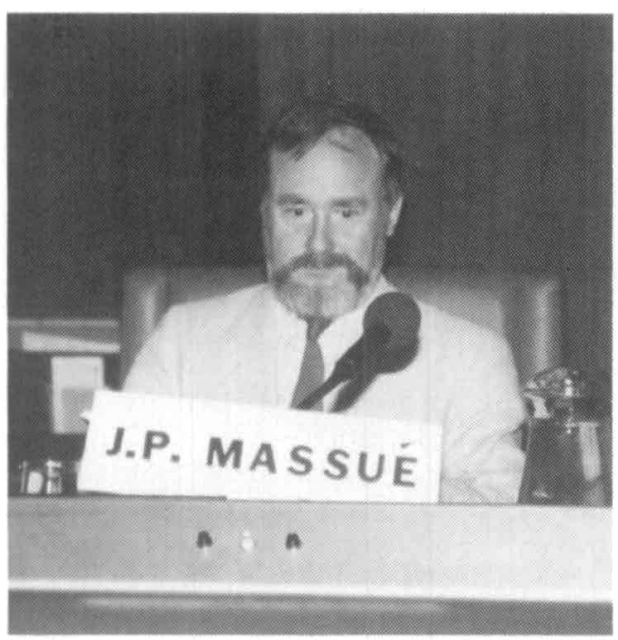

J. P. Massué, Scientific Advisor, The Parliamentary Assembly of the Council of Europe, welcomes E-MRS participants.

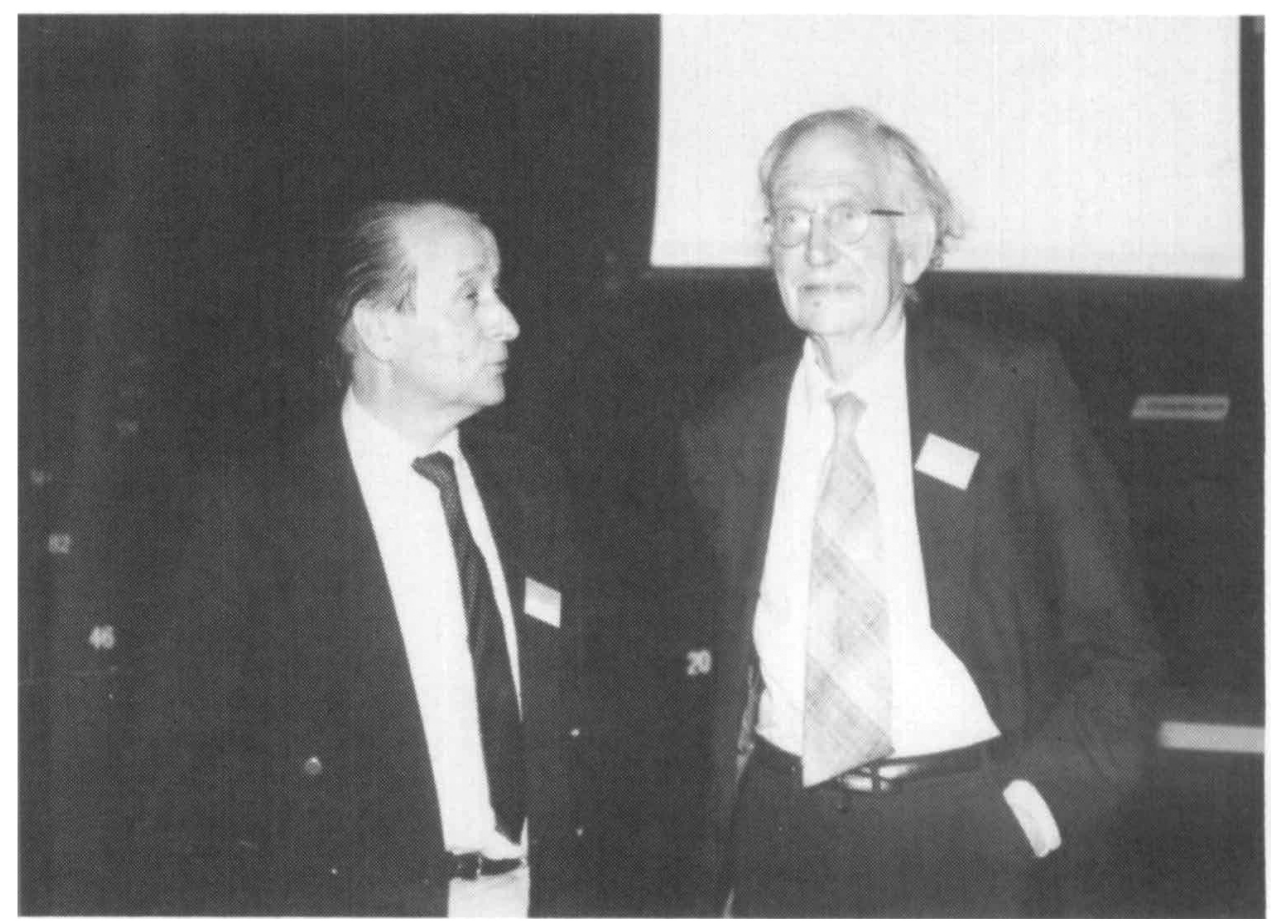

P. Siffert (left), Interim President of E-MRS greets Nobel Laureate Nevill Mott before plenary session begins.

isms. Recent work using isotopically enriched oxygen-18 has shown that, for dryoxygen oxidation of silicon, the oxygen moves through the film to the interface with silicon where new oxide is formed without exchange with the oxygen already bound in oxide. Water vapor, on the other hand, moves through the oxide so as to exchange with extant oxygen. Precise transport mechanisms in this case are still unclear.

Mott proposes that silanol groups formed with four silicon atoms and an $\mathrm{OH}$ group are the transporting species. He reports that several ancilliary mechanisms and observations have been proposed involving, for example, initial oxidation in the stoichiometry $\mathrm{SiO}$ rather than $\mathrm{SiO}_{2}$, a strong role for electron transport in the thin film stage, the existence of a variety of amorphous $\mathrm{SiO}_{2}$ structures, the importance of strain in oxide layers as a result of either volume change or misfit with silicon, and an additional model, due to Jim Phillips of Bell Labs, which contends that the so-called a morphous $\mathrm{SiO}_{2}$ is actually composed of 50 A crystobalite microcrystals with a high degree of twinning.

Mott then acting as session chairman introduced subsequent speakers. His talk was followed by two technical presentations, the first by D.T.J. Hurle concerning the mathematical relations used to describe crystal growth. Hurle discussed the analytical modeling of crystal growth processes and pointed out the various approximations involved in approaches currently used.
His presentation concent rated on Czochralski growth of crystals in which a rotating disk is used so that not only thermal and compositional questions arise, but momentum and kinematic viscosity factors enter. Hurle emphasized that even when using the Onsager coefficients in a first-order perturbation expansion, nonlinearity still enters the analysis unless an additional assumption is made. The perturbation approach initially assumes that all gradients such as compositional and thermal are in order to maintain a linear theory actually

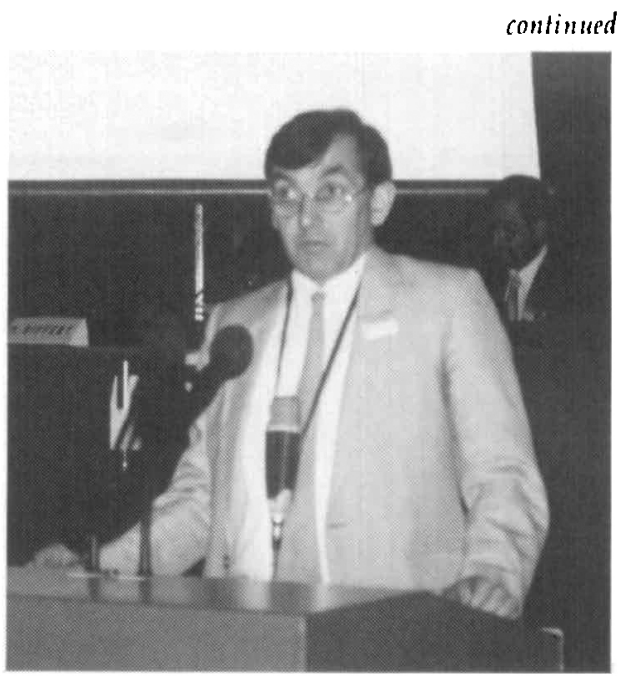

D.T.J. Hurle lectures on crystal growth at E-MRS plenary session. small. However, the additional assumption 
involves the smallness of the change in the system from the initial global thermal equilibrium reference state.

The next technical presentation was provided by J. Vannimenus, who described the concept and applications in materials science of fractals. Also included in this session were two speakers provided by the European Community (EEC) in Brussels. P. Malinverni explained ESPRIT (European Strategic Program for Research and Development in Information Technology) and indicated its progress to be substantial. Its emphasis is on promoting precompetitive transnational industrial $R \& D$ cooperations. Malinverni pointed out that a primary problem was the current lack of interEuropean compatibility in the various technical systems that information technology requires. A goal of ESPRIT is to create a much greater degree of uniformity in that regard. $M$. Konidaris from the EEC then described the program entitled "Research for Advanced Communications Europe" (RACE). This is similar to a high technology based program being pursued by the EEC specifically for advanced communications. To some extent it appears to be a subset of information technology since both are strongly based on advances in microelectronics.

Following the plenary session, participants dispersed to attend the five individual symposia technical sessions. Several of these will publish proceedings with Les Editions de Physique as in the past. A short summary of Symposium B (Laser Processing and Diagnostics) can be found elsewhere in this issue

On Wednesday evening, June 18, nearly 40 delegates to the governing committee of E-MRS met at the PLM Hotel in Strasbourg. Much discussion centered on the current success of 1986 symposia and appropriate topics for future meetings. It was also noted that the upcoming summer school sponsored by E-MRS on Semiconductor Surface and Interface Problems was oversubscribed.

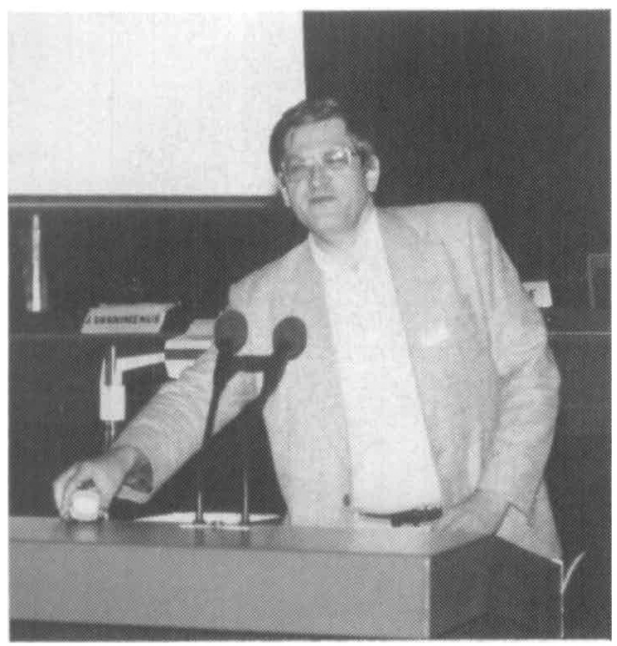

J. Vannimenus prepares to explain fractals to the E-MRS plenary session audience.
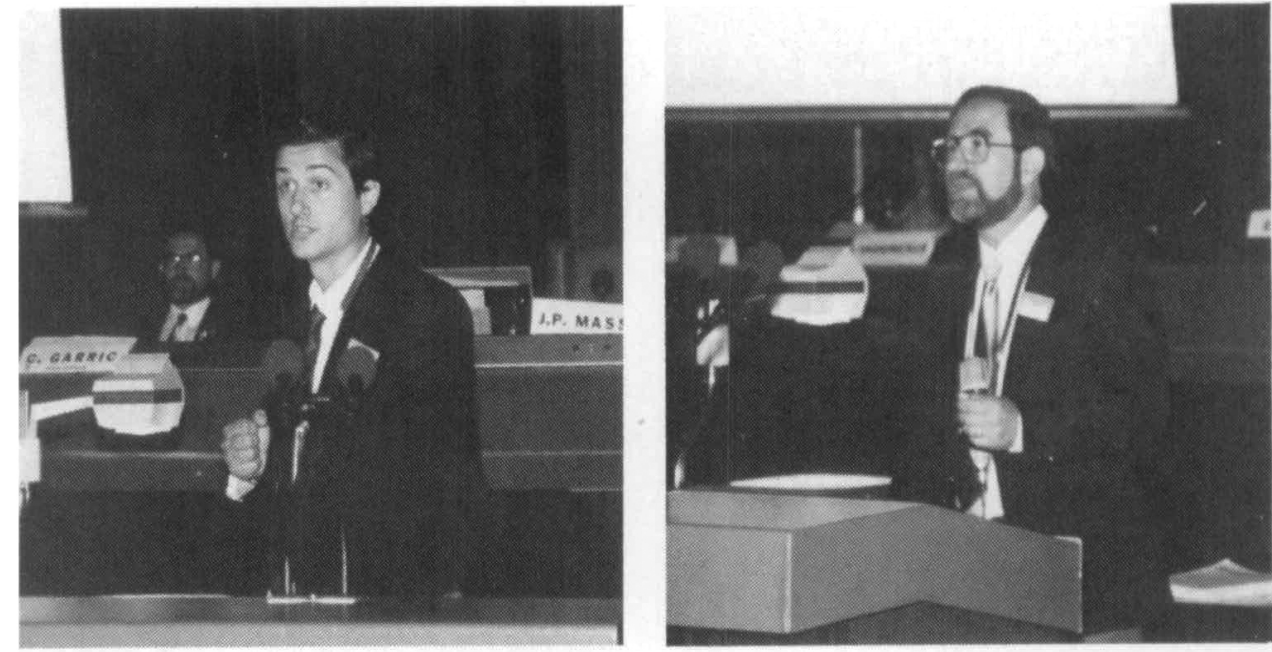

P. Malinverni of the EEC describes the ESPRIT program.

In other business, E-MRS agreed to act as a cooperating society for an MRS cosponsored meeting in Tokyo in June 1988. This meeting will include the IBMM ' 88 Conference. E-MRS also agreed to cosponsor a six-symposia meeting on Materials Processing and Property Control planned by the Institute of Metals (UK) for May 11-15, 1987. The IOM was represented at the E-MRS by Professor G. Chadwick (Southampton).

The committee also addressed issues concerning the appropriate E-MRS advisory role in relation to the European Network and European Science Foundation thrusts in materials research and heard a brief proposal for cooperation from the president of the Materials Society of Portugal. Subcommittees were established to handle matters concerning program topics for E-MRS meetings and financial support for E-MRS symposia. The Materials Research Society (U.S.) was represented at the meeting by R.P.H. Chang, D.J. Ehrlich, and E.N. Kaufmann.

\section{Laser Processing and Diagnostics}

Symposium B at the E-MRS Spring Meeting June 16, 1986 covered "Laser Processing and Diagnostics" and lasted for three days. It was organized by Dieter Bäuerle (Linz), Karl Kompa (Garching), and Lucien Laude (Mons). The eight-session symposium covered topics ranging from the fundamentals of laser-molecule-surface interactions to laser-induced deposition, surface modification, alloying, etching, and laser machining. The main topics were reviewed by invited speakers, including:

H. Walther (Garching), "Laser Investigations of the Dynamics of Molecule Surface Interaction"

R. B. Jackman (Southampton), "PhotonEnhanced Etching and Metalization Phenomena: Surface Studies"

D. Ehrlich (Lexington), "Recent Microelectronics Applications by Laser Direct
M. Konidaris of the EEC details the RACE rationale.

Writing and Excimer Projection"

H. Schroder (Garching), "Photochemistry of Surface Metalization with Excimer Lasers"

H. H. Gilgen (New York), "Hybrid Deposition of Refractory Metals"

K. Piglmayer (Linz), "Temperature Distributions in Pyrolytic Laser Induced Chemical Processing"

G. Auvert (Grenoble), "Microelectronic Films Fabrication by Chemical Laser Processing"

M. Musci (Milan), " $\mathrm{CO}_{2}$ Laser-Assisted Deposition of Amorphous Semiconductors"

$M$. von Allmen (Bern), "Nanosecond Metallurgy"

S. Metev (Sofia),"Laser Plasma Synthesis of Thin Films Polycomponent Material"

J. Fröhlingsdorf (Jülich),"A morphization of Ga by Laser Quenching"

These presentations demonstrated the widespread evolution of the field in Europe together with its potential uses in science and industry. One particular session was dedicated to laser-induced oxidation. It was jointly organized with Symposium D (Dielectric Layers in Semiconductors: Novel Technologies and Devices). For this joint session the speakers were:

I. N. Mihailescu (Bucharest), "Surface Studies with Laser with Application to Laser Material Processing"

I. W. Boyd (London), "Photoformation of Silicon Dielectrics"

R. Andrew (Mons), "Kinetics of Oxidation of Metals by CW Argon Ion Laser Irradiation"

State-of-the-art activities were further outlined by very recent results presented in both oral and poster contributions. The main emphasis was on understanding the basic physical and chemical microscopic mechanisms. The great variety of actual and potential applications is increasing by capturing the interest of technologists and manufacturers. This further increases the fascination of this young and rapidly expanding field.

continued 


\section{Dielectric Layers in Semiconductors}

At the E-MRS Spring Meeting (June 16, 1986), Symposium D on "Dielectric Layers in Semiconductors: Novel Technologies and Devices" was conceived as a forum to exchange views between people working on fundamental processes involved in the formation and characterization of dielectric layers and their a pplication in different steps of electronic device fabrication. Organized by G.G. Bentini (LAMEL, Bologna), E. Fogarassy (CNRS, Strasbourg), and A. Golanski (CNET, Grenoble), the symposium included 17 invited presentations and 30 contributed oral and poster presentations during $21 / 2$ days. A high point of the symposium was the common session with Symposium B on "Laser Processing and Diagnostics."

The plenary session was particularly honored to have a presentation by Professor Sir Nevill F. Mott on some basic problems related to the oxidation of silicon.

The first session of this symposium was devoted to fundamentals of growth and deposition of dielectric films. Three interesting invited presentations were given during the first session by $G$. Demazeau (University of Bordeaux, France) on "Oxidation of Monocrystalline Silicon in Dry Oxygen at High Pressures," by F. Gaspard (University of Grenoble, France) on "Progress in Anodic Oxidation of the Silicon," and by R.P.H. Chang (Northwestern University) on "Indirect Plasma Assisted Deposition of Dielectric Films and Device Application."

The second part of the first session dealt with the physical properties and chemical

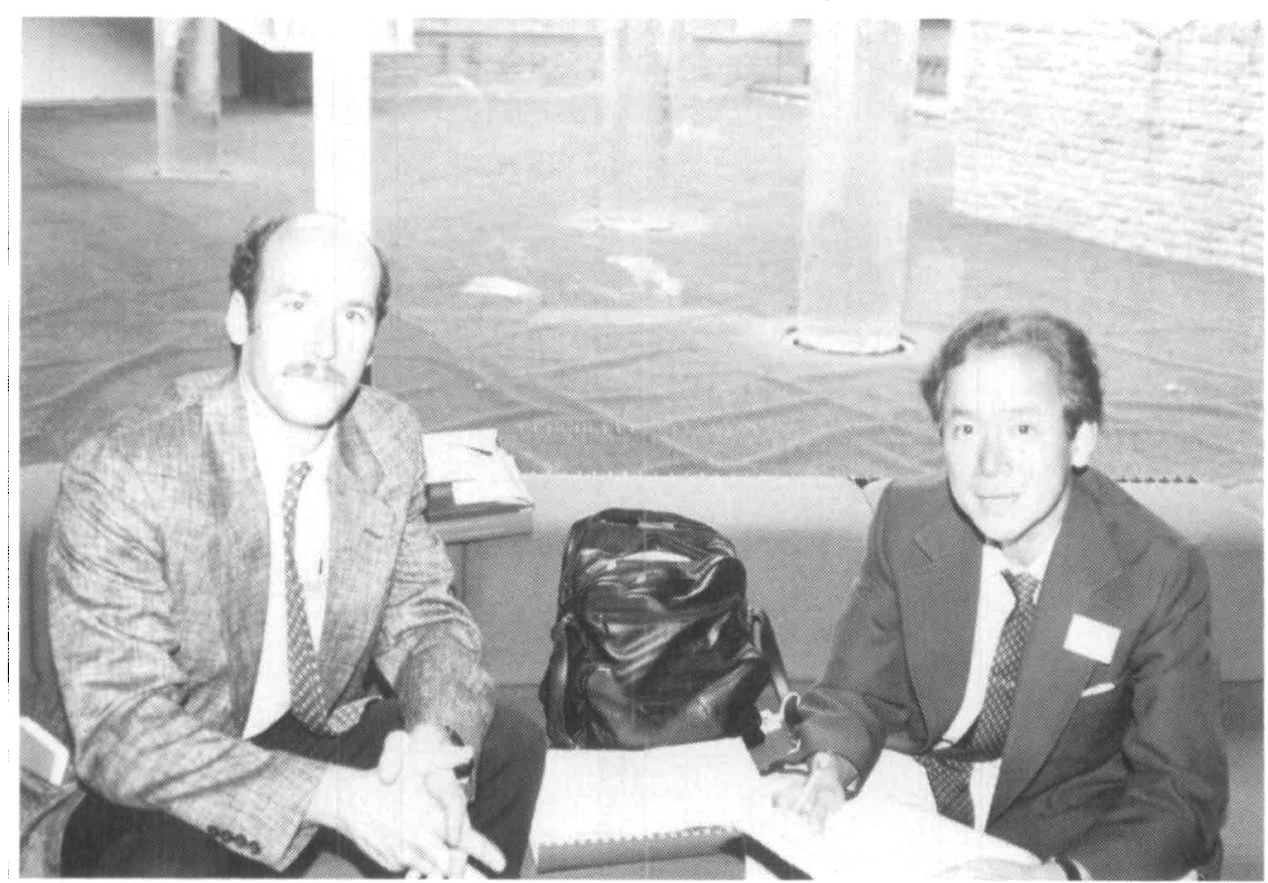

U.S. representative to the MRS International Committee, Dan Ehrlich (left)(Lincoln Labs), confers with Van Tran Nguyen (CNET, Grenoble), who is in charge of E-MRS relations with the United States. (Linz), and L. Laude (Mons). characterization of oxygen and/or nitrogen ion-implanted and high-temperature annealed silicon-on-insulator (SOI) material. Four invited papers, four orally presented contributed papers, and two contributed poster papers focused on this subject: $M$. Bruel (LETI/IRDI, France) and G.K. Celler (AT\&T Bell Laboratories) presented detailed cross-sectional TEM and RBS studies of the implanted oxygen redistribution and the SOI structure formation during high temperature treatments at $1300^{\circ} \mathrm{C}$ and $1405^{\circ} \mathrm{C}$, respectively. In the latter approach

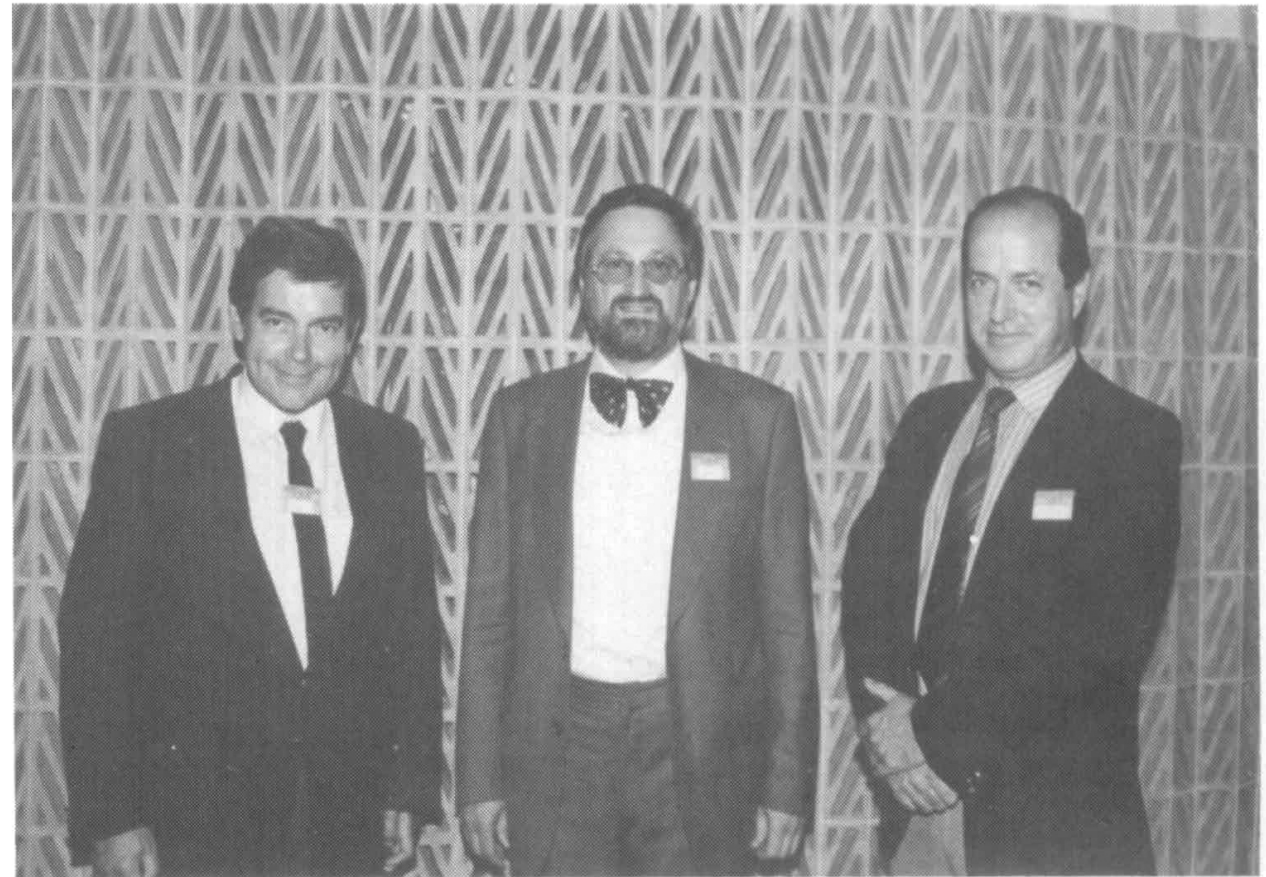

Chairmen of E-MRS Symposium B (left to right): K.L. Kompa (Garching), D. Bäuerle

the back side of the Si wa fer was held at the melt temperature of silicon $\left(1412^{\circ} \mathrm{C}\right)$. The ability of both approaches to process fullsize $(100 \mathrm{~mm})$ wafers and obtain a high quality single-crystalline Si film and abrupt $\mathrm{Si} / \mathrm{SiO}_{2}$ interfaces represents an exciting and promising development in high-speed CMOS circuits and radiation-hardened devices.

Both materials and processing issues were treated during the symposium. The influence of oxygen implantation conditions on the electrical properties of the Si overlay was discussed by A. Golanski (CNET Grenoble, France). A notable feature of the data was that the density of the oxygenrelated donor centers observed after a high-temperature $\left(1300^{\circ} \mathrm{C}\right)$ annealing is related to the substrate temperature during oxygen implantation and attains a minimum value for temperature close to $600^{\circ} \mathrm{C}$.

In Session V, M. Guerra (Eaton Corp.) discussed optimized oxygen implantation conditions which allow the directional effects occurring during oxygen implantation at elevated temperatures to be minimized. D.P. Vu (CNET Grenoble, France) presented recent results in SOI material characterization using MOS transistors fabricated within the material. Depletion-mode and enhancement-mode transistors were used for electrical evaluation (carrier concentration [electrons] and electron mobility profiles) of the Si overlayer and of the $\mathrm{Si} / \mathrm{SiO}_{2}$ interfaces. The technique may be used to test the suitability of the SOI material for device production. The depth distribution of Hall mobility and carrier concentration within the Si overlayer of the oxygen-implanted SOl, measured using p-type MOS-Hall devices were described

continued 


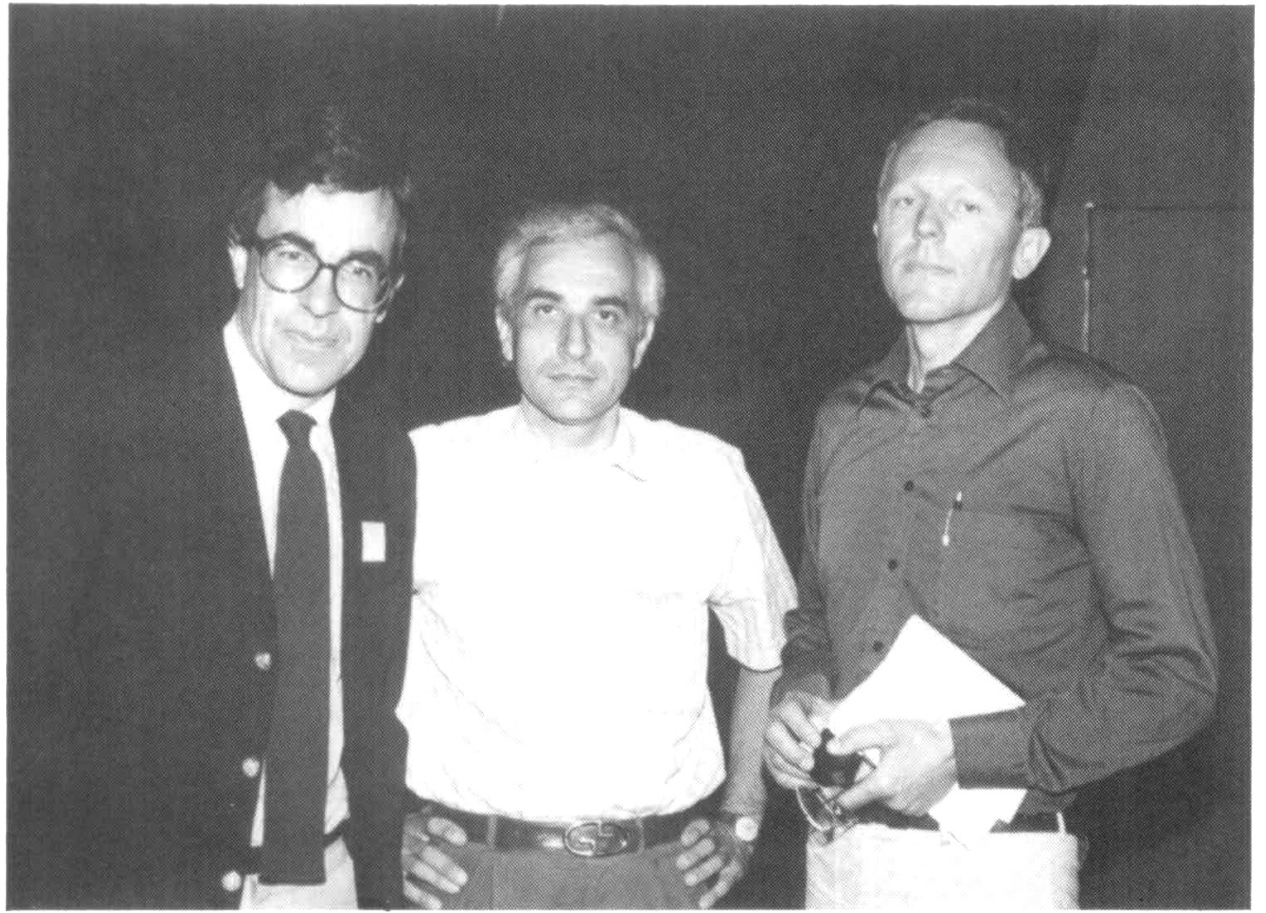

Chairmen of E-MRS Symposium D (left to right): E. Fogarassy (CNRS), G.G. Bentini (CNR-Institute LAMEL, Bologna), and A. Golanski (CNET-CNS, Grenoble).

by S. Cristoloveanu (ENSERG, France).

Finally, an overview of the most promising approaches to SOI substrate preparation with respect to the material properties of the Si overlayer was presented in Session $\mathrm{V}$ by S. Partridge (GEC Research, UK). It appears that although considerable attention has been focused on the oxygenimplanted SOI material with very interesting and promising results and the successful development of implantation machines, there is an obvious need for better
The works presented in this session focused on the mechanisms of laser-induced oxidation of silicon and compound semiconductors occurring as a consequence of photolitic and/or pyrolitic excitation. In general, these nonconventional laser-assisted techniques have demonstrated the capability of offering new approaches for largearea low-temperature processing and/or for ultrafast growth of reacted layers if high laser fluences are used.

Three invited papers were especially devoted to this area: "Photoformation of Silicon Dielectrics" by I.W. Boyd (University College, London), "Loss and Incorporation Mechanisms During Pulsed Laser Irradiation of Silicon in Gaseous Atmosphere" by C. Cohen (University of Paris, VI), and "Surface Studies with Laser with Applications to Laser Material Processing" by I.N. Mihailescu (Institute of Physics, Bucharest, Romania). The oxidation mechanism of metals induced by laser irradiation was reported by $R$. Andrew (University of Mons, Belgium), in "Kinetics of Oxidation of Metals by CW Argon Ion Laser Irradiation."

The third session dealt with $\mathrm{Si}-\mathrm{SiO}_{2}$ interface and thin oxide properties. Very good reviews were presented by $P$. Balk (Aachen, FRG) on the "The $\mathrm{Si}_{-} \mathrm{SiO}_{2}$ System and Its Interface," by M. Severi (LAMEL, Italy) on "Gate Dielectrics for VLSI-MOS Technology," and by M. Heyns (IMEC, Belgium) on "Thin Gate Insulators for VLSI." The fourth session focused on compound semiconductors with a particulary interesting talk by H.L. Hartnagel (Darmstadt, FRG) on "Oxides and Other Dielectric Surface Layers on Compound Semiconductors."

\section{Proceedings of the 1984 E-MRS Meeting}

Poly-Micro-Crystalline and Amorphous Semiconductors

Amorphous Metals and Non-equilibrium Processing

Induced Defects in Insulators
MRS Members: $\$ 52.00$

MRS Members: $\$ 40.00$

MRS Members: $\$ 34.00$

\section{Send payment to Materials Research Society}

9800 McKnight Road, Suite 327, Pittsburgh, PA 15237; telephone (412) 367-3012. 